\title{
Analytical Study of Design Parameters of Co-Axial Antenna for Microwave Ablation Therapy using COMSOL Multiphysics
}

\author{
Amninder Kaur \\ Associate Professor \\ Dronacharya College of Engg, \\ Gurgaon
}

\author{
Neeru Malhotra \\ HOD ECE \\ DAV Institute of \\ Engg.\& Technology \\ Bhavesh \\ B.Tech. Student \\ Dronacharya College of Engg, Gurgaon
}

\author{
Saniya Puri \\ B.Tech. Student \\ Dronacharya College of Engg, \\ Gurgaon
}

\begin{abstract}
Hyperthermia is in the interest of both, clinical and research oncologists, because of its properties to directly produce permanent damages of the treated tumors and to elicit important immunological responses against cancer cells by changing their immunogenicity. Microwave ablation (MWA) is used in the treatment of primary and secondary tumors of the liver. The purpose of this research is to study the fundamental physical mechanisms of microwave ablation of liver tissue and to utilize the results to improve microwave tumor ablation systems. In this research it has been discussed how advanced electromagnetic numerical techniques can be utilized to design the antennas for realistic human body environment. Different mathematical methods may be employed for the computation of power distributions in biological structures irradiated by a source of electromagnetic radiation.
\end{abstract}

\section{Keywords}

Heptocellular Carcinoma (HCC), Microwave coagulation therapy (MCT), Radiofrequency ablation (RFC).

\section{INTRODUCTION}

Cancer is one of the most common malignant tumors with an estimated 1,000,000 worldwide deaths per year. Persistent or recurrent liver disease is the major cause of both morbidity and mortality in patients with Heptocellular Carcinoma (HCC) [1].In comparison to other body parts, the liver is the most common site of distant metastasis of colorectal cancer. Nearly half of the patients suffering from colorectal cancer ultimately develop liver involvement during their diseases. As per the study conducted nearly 150,000 new cases of colorectal cancer will be diagnosed in the US each year with 57,000 deaths [2].

\subsection{Primary Liver Cancer}

Primary liver cancer, known as Heptocellular Carcinoma(HCC) accounts for $80 \%-90 \%$ of all liver cancers. Male population is more prone to this disease and it occurs more often in men than women, and occurs mostly in age group of 5- 60 years old. The disease is becoming more common in the West recently due to an increase in hepatitis cases where as it was more prevalent in parts of Africa and Asia . The factors which may lead to this disease are chronic liver disease, viral, and food toxins; tough exact root cause is still under investigation.

\subsection{Secondary Liver Cancer}

Metastasis is the movement or spreading of cancer cells from one organ or tissue to another. The liver is a common site of metastasis from the gastrointestinal tract and from a variety of organs such as the lung, breast, colon and rectum. The liver is frequently involved since it receives blood from the abdominal organs via portal vein [3].

\section{TREATMENT OF LIVER CANCER}

The disease for at-risk population can be controlled with vaccines for both Hepatitis B \& C, where for extirpation of tumor one must undergo the proven techniques for csncer ie chemothearpy and radiation therapy. Surgical resection is the gold standard for the treatment of patients with respectable isolated hepatic metastases with $40 \% 5$ year and $26 \% 10$ years survival. Cancer enters the bloodstream or lymphatic channels, travel to the liver and grow independently. Ablative treatments have started to become viable alternative methods to treat patients who cannot be treated by surgery. Such ablative treatments include cryoablation, radiofrequency ablation (RFA), microwave ablation (MWA) or also called microwave coagulation therapy (MCT), and ethanol ablation, etc. RFA has been used in clinical operations in the USA for years while MWA is still undergoing major improvements and is actively researched. Clinical trials for microwave liver ablation have been carried out in Asian countries.

\section{HYPERTHERMIA PLANNING}

TREATMENT

The goal of hyperthermia treatment Planning is to define the power deposition pattern required to produce the necessary results-raising the temperature of the tumor to therapeutic levels while keeping the amount of normal tissue damage to acceptable minimum. There is a difference between the ideal temperature distribution, in which the entire tumor and none of the normal tissue is raised to therapeutic temperatures, and clinically practical temperature distributions. The goal is to maximize the volume of tumor raised to therapeutic temperatures while limiting the volume of normal tissue being damaged to a clinically acceptable value [4]. It is difficult to achieve a homogeneous temperature distribution since in most clinical hyperthermia cases heterogeneous temperature distributions are achieved for varying times.

Simulations presented here can be used for the treatment of liver cancer and focus on the analysis of control of radiation patterns and power deposited within human tissue. Finite 
Element Method is an efficient technique used for performing analysis of complex structures allowing the flexibility in changing the shape of the antenna. A finite element model is developed to determine the absorbed power and the temperature distribution surrounding the single coaxial antenna. In hyperthermic oncology, cancer is treated by applying localized heating to the tumor tissue, often in combination with chemotherapy or radiotherapy [5]. Some of the challenges associated with the selective heating of deepseated tumors without damaging surrounding tissue are:

Ç Control of he at ing powe r Ç Design a nd place me n t of

\section{MICROWAVE TISSUE ABALATION FUNDAMENTALS}

The basic principle of microwave hepatic ablation is to apply microwave power to the liver tissue through the microwave applicator. The power of the EM wave is absorbed by the liver tissue and heats the tissue. Liver tissue is destroyed after the tissue is heated to a high enough for a long enough time [6]. Fig. 1 shows basic devices to perform a MWA are a microwave generator, a microwave applicator, and a section of flexible coaxial cable to connect the antenna to the microwave generator. Ultrasound scanners are often used in the MWA procedures to guide the placement of the applicator [7]. Fiber-optic thermometers can be used to measure tissue temperature. MRI scanners can be used to examine lesion size after the procedures.

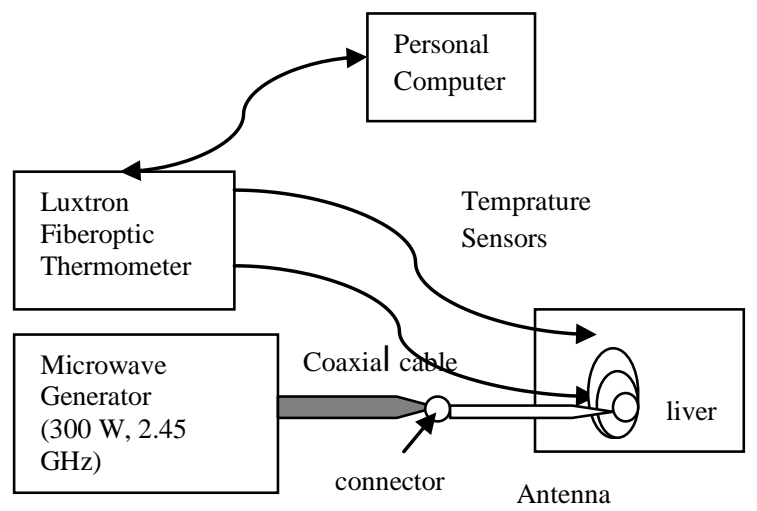

Fig.1 Schematic of experimental setup of microwave liver tissue ablation. For a clinical procedure, the Luxtron fiber-optic thermometer and the temperature probes are not used.

In a clinical MWA procedure, position of the tumor is determined in advance with medical imaging devices, including MRI, CT or ultrasound devices. A MWA probe is placed into the tumor with an open surgery or a percutaneous procedure, guided by ultrasound or other medical imaging device. The probe is connected to the microwave power generator. Microwave power level and heating duration are selected in advance according to the shape and size of the tumor. Microwave power is then applied for the selected duration [8].

\section{SELECTING FINITE ELEMENT METHOD FOR PRESENT WORK}

In this work COMSOL version 3.3 is used as primary computer simulation tools. FEMLAB is a commercial software package, which solves partial differential equations using the finite element method (FEM). COMSOL can be used standalone. It can also be fully integrated with MA T L A B Ì . My b a s i c E M mo d e l slot antenna EM model from COMSOL. The EM models are powerful tools to study the antennas static performance.

a $n$ d. s pa t i a l d i s t r i b u t i. o n $t$ e mpinite Element Method $\mathrm{e}_{\mathrm{n}}$ an $\mathrm{s}$ ffficient technique used for performing analysis of complex structures allowing the flexibility in changing the shape of the antenna. This method consists of representing a given domain, however, complex it may be by geometrically simple shapes over which the approximation functions can be systematically derived [9]. The Finite Element Method not only overcomes the shortcomings of the traditional analytical and numerical methods but it also endowed with the features of an effective computational technique. FEM models can provide users with quick, accurate solutions to multiple systems of differential equations.

\section{ANALYSIS USING FEM MODEL DEFINATION}

Microwave coagulation therapy is one such technique where a thin microwave antenna is inserted into the tumor. The microwaves heat up the tumor, producing a coagulated region where the cancer cells are killed. The purpose of this model is to compute the temperature field, radiation field and the specific absorption rate (SAR) in the liver tissue, when using a thin coaxial slot antenna used in microwave coagulation therapy. The temperature distribution in the tissue is computed using the bio heat equation. The antenna geometry is shown in Figure 2.

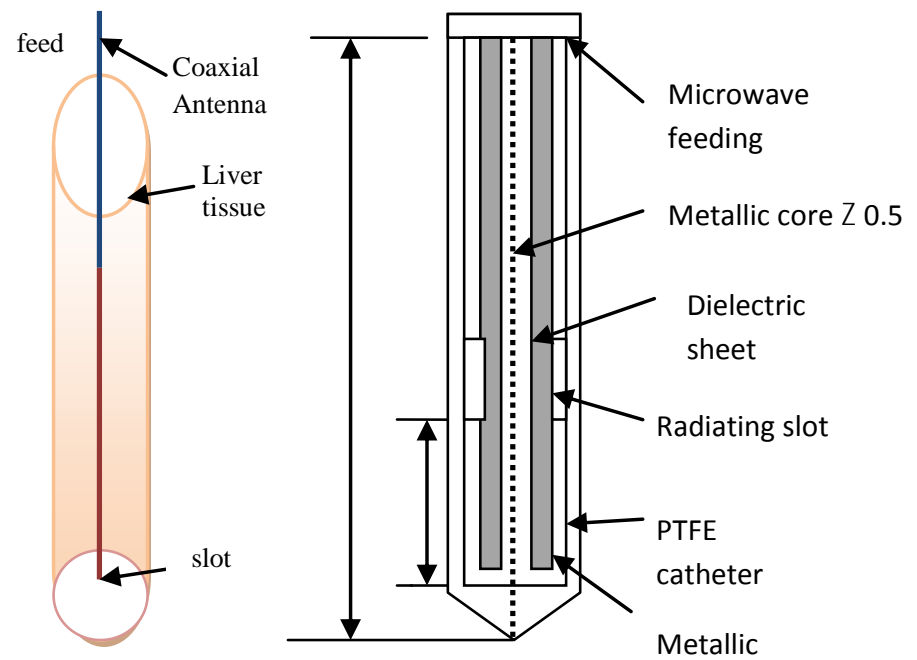

Fig. 2. The antenna geometry is shown w a s 
Table 1: Dimensions and material properties of the Coaxial Slot Antenna

\begin{tabular}{|l|c|}
\hline \multicolumn{1}{|c|}{ PROPERTY } & VALUE \\
\hline Diameter of the central conductor & $0.29 \mathrm{~mm}$ \\
\hline Inner diameter of the outer conductor & $0.94 \mathrm{~mm}$ \\
\hline Outer diameter of the outer conductor & $1.19 \mathrm{~mm}$ \\
\hline Diameter of catheter & $1.79 \mathrm{~mm}$ \\
\hline
\end{tabular}

It consists of a thin coaxial cable with a $1 \mathrm{~mm}$ wide ring shaped slot cut on the outer conductor $5 \mathrm{~mm}$ from the shortcircuited tip. For hygienic purposes, the antenna is enclosed in a sleeve (catheter) made of PTFE (polytetrafluoroethylene). Material data and geometrical dimensions are given in the table below. The antenna is operated at $2.45 \mathrm{GHz}$, a frequency widely used in microwave coagulation therapy [10]

Antenna geometry parameters, the slot spacing, choke offset, choke length, etc, were chosen based on the effective wavelength in liver tissue at $2.45 \mathrm{GHz}$, which was calculated using

$$
v_{\text {eff }}=\mathrm{c} / \mathrm{f} \sqrt{ } \gamma_{\mathrm{r}}[\mathrm{m}]
$$

where $c$ is the speed of light in free space $(\mathrm{m} / \mathrm{s}), f$ is the operating frequency of the microwave generator $(2.45 \mathrm{GHz})$, and $\gamma_{\mathrm{r}}=44.4$ is the relative permittivity of bovine liver tissue at the operating frequency; this yielded $18.4 \mathrm{~mm}$ for the effective wavelength [11].

Table 2.shows the frequency independent material parameters used in this model

\begin{tabular}{|l|c|}
\hline \multicolumn{1}{|c|}{ Name } & Expression \\
\hline k_liver & 0.56 \\
\hline rho_blood & 1000 \\
\hline c_blood & 3639 \\
\hline omega_blood & $3.6 \mathrm{e}-3$ \\
\hline T_blood & 310 \\
\hline epsilon0 & $8.8542 \mathrm{e}-12$ \\
\hline mu0 & $4 *$ pi*1e-7 \\
\hline eps_diel & 2.03 \\
\hline eps_cat & 2.6 \\
\hline eps_liver & 43.03 \\
\hline sig_liver & 1.69 \\
\hline
\end{tabular}

However, because the catheter and its thickness also affect the optimal geometry and performance of the antenna, equation only provides a very crude approximation for the design. Tip length and catheter thickness were adjusted to achieve resonance. For simplicity and to eliminate numerical error, the inner and outer conductors of the antenna are modeled using perfect electric conductor (PEC) boundary conditions. Lowreflecting boundary conditions are used along the model boundaries to prevent reflection artifacts and axial symmetry boundary conditions were also employed along the axis of rotation [12].

\section{BASIC COMPUTER SIMULATIONS FOR MWA}

In this work COMSOL version 3.3 is used as primary computer simulation tools. FEMLAB is a commercial software package, which solves partial differential equations using the finite element method (FEM). COMSOL can be used standalone. It can also be fully integrated with MATLABЯ. My basic EM model was adapted from a basic slot antenna EM model from COMSOL. I learned how to define the power source in my axial-symmetric models from the COMSOL model. The EM models are powerful tools for me to study the antennas static performance. I can create new antenna designs, modify the antenna geometrical parameters, change the dielectric properties of the medium or the dielectric material of the coaxial cable, etc. I can then observe the antenna performance changes instantly after I modify the model parameters, and to select the optimal antenna parameters according to my predetermined rules.

\section{RESULTS}

After the EM model is solved, the post processing features in COMSOL can be used to analyze the solution by plotting graphs for all field variables. We made the slot length 1.0 $\mathrm{mm}$ and height $5 \mathrm{~mm}$ from tip which produced the following iterations for various values of Power input $1 \mathrm{~W}: 5 \mathrm{~W}: 10 \mathrm{~W}$. The different power values gave different results for maximum power transfer \& maximum \& temperature achieved rate of change of temperature.

Simulation shows the temperature in the liver tissue shows the resulting steady-state temperature distribution in the liver tissue for an input microwave power of $10 \mathrm{~W}$. The temperature is highest near the antenna. It then decreases with the distance from the antenna and reaches $310 \mathrm{~K}$ closer to the outer boundaries of the computational domain. The perfusion of relatively cold blood seems to limit the extent of the area that is heated.

\subsection{Result for 1W Power}

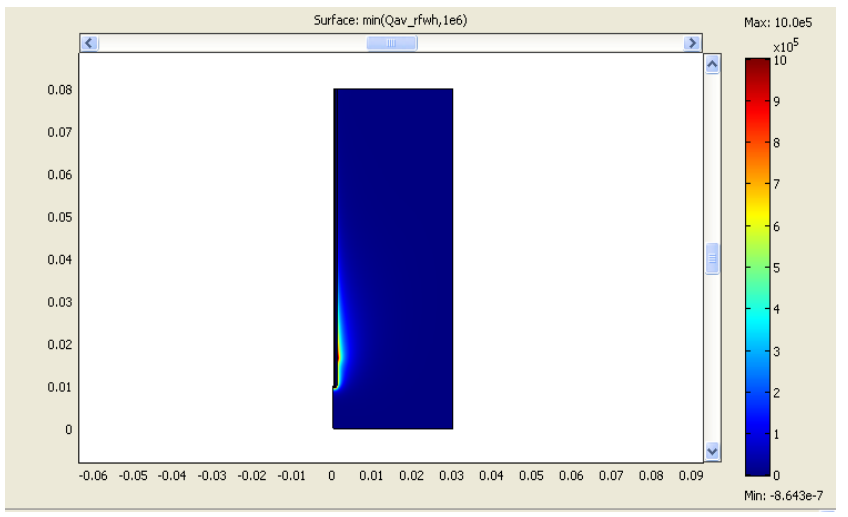

Fig.3.Simulation results: Temperature in the liver tissue at power 1W 


\subsection{Result for $5 \mathrm{~W}$ power:}

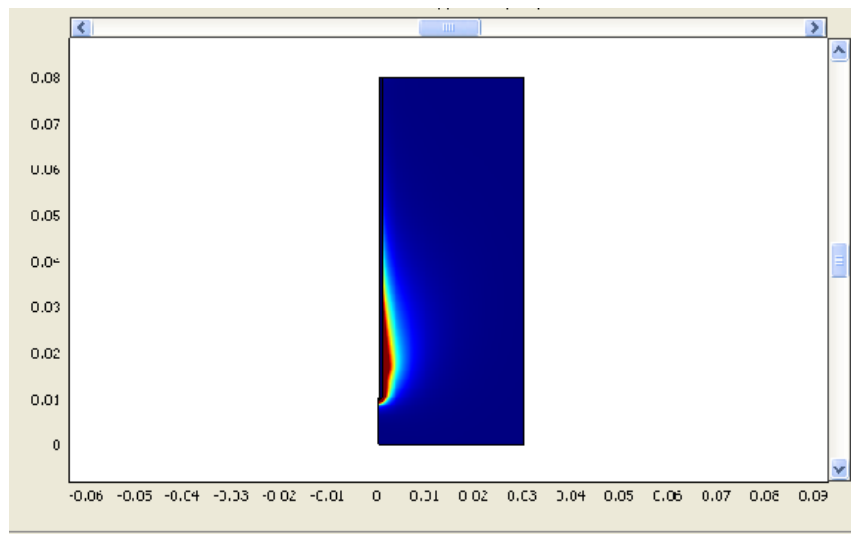

Fig.4.Simulation shows the temperature in the liver tissue at power $5 \mathrm{~W}$

\subsection{Result for $10 \mathrm{~W}$ power:}

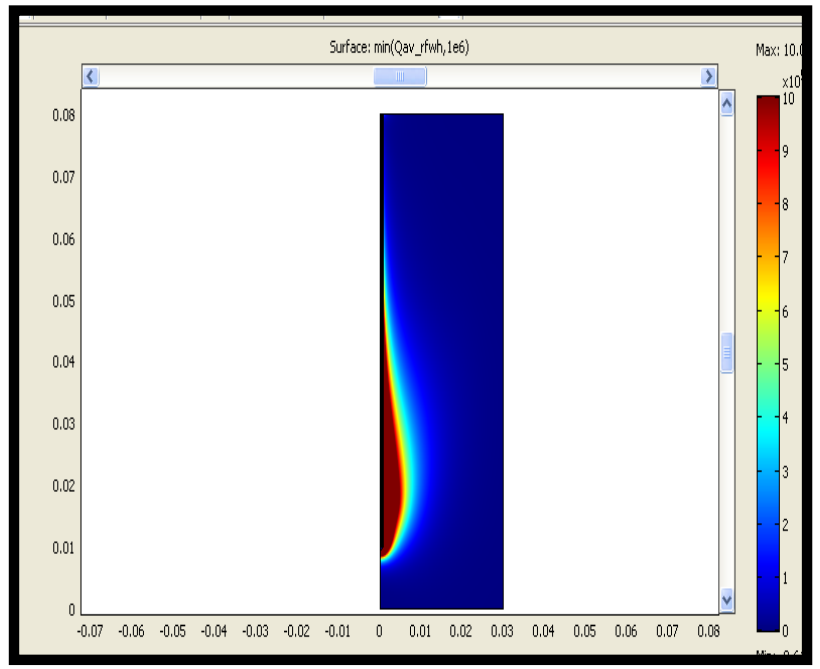

Fig. 5. Simulation Results: the temperature in the liver tissue at power $10 \mathrm{~W}$

The study was carried out with the conscious aim of optimizing the antenna power for various sizes of tumors. The plot for temperature distribution provides a suitable platform to conclude that tumors of different shape and sizes can be cured by using different power input.

Table 3: Coordinate of peak temp. v/s input power

\begin{tabular}{|c|c|c|c|}
\hline $\begin{array}{c}\text { Iterations } \\
\text { Antenna } \\
\text { Parameter }\end{array}$ & $\mathbf{1}$ & $\mathbf{2}$ & $\mathbf{3}$ \\
\hline Power Input & $1 \mathrm{~W}$ & $5 \mathrm{~W}$ & $10 \mathrm{~W}$ \\
\hline Power Transferred & $.93 \mathrm{~W}$ & 4.68 & $9.36 \mathrm{~W}$ \\
\hline $\begin{array}{c}\mathrm{Z}_{\mathrm{p}(\text { Coorinate }} \text { for peak } \\
\text { temp. })\end{array}$ & $\begin{array}{c}3.6 \\
\mathrm{~mm}\end{array}$ & 15 & 27 \\
\hline
\end{tabular}

\section{CONCLUSION}

To summarize, it can be seen from the compilation of data that the as the power is increased there is sufficient increase in the coordinate of peak temperature i.e. the net effective area under the influence of high temperature had been increased. This may have other implication such that damage of healthy tissues, irritation to the patient and burning of skin. So the comprise between the increase in power should be cautiously balanced with the effects to the surroundings.

\section{REFERENCES}

[ 1 ] J e ma l, R. S i e g e l, E. Wa r d, A cancer journal $\mathrm{f}$ o $\mathrm{r}$ c l i n i c i a $\mathrm{n} \mathrm{s}$. C a n American cancer society, 2007.

[ 2 ]. E. L. Ma y e r, H. J. Bu r s t me t a s t a t i c , DanaeFarbertCanceraImstitute, $r$ ô Brigham and Women's Hospital, Harvard Medical School, 44 Binney Street, Boston, MA 02115,SA.(2007).

[3]. S. J a c o b s e n, P. S t a u e-band ó C a $n$ radiometrictemperature monitoring during hyperthermia treatment of chestwall re-currence of breast cancer using a dual-mo d e $t$ r a n s c e iPhys. Meg. Biøl.p5 911-928, 2007.

[4]M. Danta, E. B a r n e s, and G. Dus a nd d i a g n o s i s of h Eup aJt o c a l u GastroenterolHepatol, 17(5):491-496, May 2005.

[5]. L. X. Liu, W. H. Zhang, and H. C. Jiang, "Current treatment for liver metastases from colorectal cancer," World J Gastroenterol, vol. 9,pp. 193-200, 2003.

[6]. C. Erce and R. W. Parks, "Interstitial ablative techniques for hepatic tumours," BrJSurg, vol. 90, pp. 272-289, 2003.

[7]. Simon CJ, Dupuy DE, Mayo-S mi t h W. W. ó Mi c $r$ a b l a t i o n : principles a nd a p pl : S69-S83, 2005.

[ 8 ] . O. P. Gand h i, J. F. De F o r method for calculation of power deposition patterns in ma g n e t i c a l l y i n d uIEEEd Tranł y p e $\mathrm{r} \mathrm{t} \mathrm{h}$ Biomed. Eng., vol. BME-31, pp. 644ñ651, 1984.

[9]. R. D. Nevels, G. D. Arndt, G. W. Raffoul, J. R. Carl and $\mathrm{P}$ a c i f i c o, ó Mi c $\mathrm{r} \rho \hat{o}$ a $\mathrm{U} \notin \mathrm{E} \mathrm{E}$ a $\mathrm{tTh} \oplus$ th Biomed. Eng., vol. 45,pp. 885-890, 1998 .

[10] .Schaubert, D. Wilton, D. Glisson, A., ótetrahedral modeling method for electromagnetic scattering by a r b i t r a r i l y s ha ped i n ho mog e n IEEE Trans. Antennas Propagat., vol 32, pp. 77-85, January 1984.

[ 11 1 $]$ ] . K W Ye e, ó Nume r i c a l s o l u Value Problems in Iso $\mathrm{t} r$ o p i c IERIF drans, ô Antenna Propagation, vol 14, 1966.

[ $\left.\begin{array}{ll}1 & 2\end{array}\right]$ ] $\quad \mathrm{P} \quad \mathrm{S}$ i l v e $\mathrm{s} \mathrm{t}$ @rder FinóteAElenGent $\mathrm{n}$ e $\mathrm{r}$ a $\mathrm{l}$ Wave-g u i d e An a l y EEEETranB. MdTg roh m, ô 4, 1969 . 